\title{
Grau de dependência de idosos hospitalizados conforme o sistema de classificação de pacientes
}

Degree of dependence of hospitalized elderly according to the patients' classification system

Grado de dependencia de ancianos hospitalizados, según el sistema de clasificación de pacientes

\section{Janete de Souza Urbanetto', Roberta Marco", Sidiclei Machado Carvalho", Marion Creutzberg', Karen Fernandes Oliveira", Tânia Bosi de Souza Magnago"II}

'Pontifícia Universidade Católica do Rio Grande do Sul, Faculdade de Enfermagem, Nutrição e Fisioterapia, Curso de Graduação em Enfermagem. Porto Alegre-RS, Brasil.

"Pontifícia Universidade Católica do Rio Grande do Sul, Hospital São Lucas. Porto Alegre-RS, Brasil.

II' Universidade Federal de Santa Maria, Curso de Enfermagem. Santa Maria-RS, Brasil.

Submissão: 19-06-2011 Aprovação: 22-01-2013

\section{RESUMO}

O objetivo do estudo foi avaliar o grau de dependência dos idosos internados, conforme o sistema de classificação de pacientes; e identificar fatores relacionados à dependência dos pacientes para o cuidado de enfermagem. Delineamento transversal, com amostra de 161 pacientes idosos avaliados no início, meio e final da hospitalização, pela escala de classificação de pacientes. Identificou-se prevalência na classificação intermediária (52,2\%); associação estatística significativa da faixa etária de 80 a 100 anos com as classes intermediária e semi-intensiva; $40 \%$ dos classificados com cuidados semi-intensivos tiveram o óbito como desfecho. O processo de envelhecimento deve determinar cuidados de enfermagem específicos para esse grupo populacional, garantindo a qualidade da assistência.

Descritores: Assistência a Idosos; Avaliação em Enfermagem; Classificação; Cuidados de Enfermagem.

\begin{abstract}
The study aimed to evaluate the degree of dependency of elderly inpatients, according to the classification system of patients, and to identify factors related to patient dependency to nursing care. Cross-sectional study with a sample of 161 elderly patients evaluated at the beginning, middle and end of hospitalization, according to the patient classification scale. It was identified a prevalence in the intermediate rating $(52.2 \%)$; statistically significant association between the ages of 80 to 100 years with the middle and semi-intensive classes; $40 \%$ of the elderly classified to the semi-intensive care had death as the outcome. The aging process must determine specific nursing care for this population group, ensuring the quality of care.
\end{abstract}

Key words: Old Age Assistance; Nursing Assessment; Classification; Nursing Cares.

\section{RESUMEN}

El objetivo del estudio fue evaluar el grado de dependencia de los ancianos hospitalizados, de acuerdo con el sistema de clasificación de pacientes, e identificar los factores relacionados con la dependencia del paciente a la atención de enfermería. Estudio transversal con una muestra de 161 pacientes de edad avanzada evaluados al inicio, mitad y final de la hospitalización, con la escala de clasificación de pacientes. Fue identificada prevalencia en la clasificación intermedia (52,2\%); se encontró asociación estadísticamente significativa entre las edades de 80 a 100 años y las clases medias y semi-intensivo; y $40 \%$ de los ancianos clasificados como de atención semi-intensiva tuvieran la muerte como resultado. El proceso de envejecimiento debe determinar los cuidados de enfermería específicos para este grupo de población, asegurando la calidad de la atención.

Palabras clave: Asistencia a los Ancianos; Evaluación en Enfermería; Clasificación; Cuidados en Enfermería. 


\section{INTRODUÇÃO}

A identificação do grau de dependência, em relação ao cuidado de enfermagem, por meio do Sistema de Classificação de Pacientes (SCP) é fundamental para o planejamento da assistência de enfermagem ao indivíduo hospitalizado e para o dimensionamento de pessoal. Discussões e investigações têm sido realizadas para a construção de um serviço de enfermagem de qualidade, com maior atenção às necessidades dos pacientes ${ }^{(1)}$.

As primeiras experiências de classificação de pacientes em relação aos cuidados de enfermagem foram desenvolvidas por Florence Nightingale, que organizava as enfermarias de modo que os mais graves fossem alocados nas proximidades das mesas das enfermeiras. Na década de 1930, o SCP começou a ser desenvolvido nos Estados Unidos e passou a ser utilizado nos hospitais. A principal finalidade era de analisar a dependência do paciente internado, estimar o tipo e a quantidade de recursos necessários para o cuidado ${ }^{(2)}$.

No Brasil, uma das primeiras autoras a tratar do assunto foi Ribeiro que, em 1972, utilizou a denominação Cuidado Progressivo dos Pacientes (CPP). Subsidiou o dimensionamento de recursos humanos em enfermagem, com o propósito de garantir uma distribuição igualitária da assistência, aumentando a produtividade e a eficiência hospitalar ${ }^{(3)}$.

Nos anos 1990 os SCPs começaram a ser desenvolvidos em diferentes especialidades e empregados nas instituições hospitalares. A recomendação para seu uso e determinação da competência do enfermeiro veio somente em 1996 (COFEN 189/1996) e atualizada em 2004 (COFEN 293/2004). Foi estabelecido que o cálculo de pessoal de enfermagem deveria ter como base a aplicação de SCP como um dos indicadores para estabelecer o perfil de cada paciente nas unidades hospitalares, as horas mínimas de ausência e a distribuição dos profissionais para cada tipo de cuidado ${ }^{(4)}$.

Perroca e Gaidzinski construíram um instrumento para classificação de pacientes baseado nas necessidades humanas básicas preconizadas por Wanda Horta e fundamentado por um extenso levantamento bibliográfico. Foram considerados 13 indicadores críticos: estado mental e nível de consciência, oxigenação, sinais vitais, nutrição e hidratação, motilidade, locomoção, cuidado corporal, eliminações, terapêutica, educação à saúde, comportamento, comunicação e integridade cutaneomucosa $^{(3)}$.

É, portanto, um processo no qual se procura identificar pacientes de acordo com a dependência de cuidado de enfermagem requerida, ou seja, baseada no grau de complexidade da assistência de enfermagem ${ }^{(5)}$. Em qualquer tipo de unidade de internação os pacientes apresentam diferentes graus de complexidade e de necessidade de cuidado. Assim, mesmo em unidades de cuidados específicos, como uma Unidade Geriátrica, tal identificação é fundamental para a organização da assistência. Tal prática insere-se no contexto das Diretrizes da Política Nacional de Saúde da Pessoa Idosa segundo a qual, em qualquer nível de atenção, é preciso oferecer cuidados sistematizados e adequados a essa população, garantindo a qualidade da atenção e pautada nos conhecimentos sobre o cuidado ao idoso - nesse caso, do idoso hospitalizado e fragilizado(6).

\section{OBJETIVO}

Este estudo teve como objetivo avaliar o grau de dependência dos idosos internados, conforme o sistema de classificação de pacientes e identificar fatores relacionados à dependência dos pacientes para o cuidado de enfermagem.

\section{MÉTODO}

Estudo com delineamento transversal, desenvolvido em uma Unidade de Internação Geriátrica do Hospital São Lucas, da PUCRS. Nesta instituição, o SCP(3) foi introduzido no ano de 2007 e aplicado, por enfermeiros, em todas as unidades de internação e emergência adulto. Para a utilização do SCP, os enfermeiros foram capacitados no ano de 2006, e periodicamente nos anos posteriores, a fim de obter-se o máximo de fidedignidade nas avaliações. Estes dados estão armazenados em um programa informatizado do próprio hospital e faz parte do sistema de informações gerenciais da Instituição.

A amostra do estudo foi composta pelos pacientes internados de maio a dezembro de 2010 e que foram avaliados pelo $\mathrm{SCP}^{(3)}$, no mínimo três vezes durante a internação nos períodos acima referidos, totalizando 161 pacientes. Como cada paciente foi avaliado três vezes, foram realizadas 483 avaliações.

Os dados foram coletados a partir das informações contidas no banco de dados, utilizando-se os registros referentes às pontuações para cada um dos 13 indicadores críticos de cuidado, assim como o escore de dependência, por paciente avaliado.

Cada indicador possui gradação de 1 a 5, apontando a intensidade crescente de complexidade, sendo o valor 1 referente ao menor nível e o valor 5 ao nível máximo de complexidade assistencial. Os valores são somados, individualmente, levando a classes de cuidados: cuidados mínimos, intermediários, semi-intensivos e intensivos ${ }^{(3)}$.

Nos cuidados mínimos (13-26 pontos) estão incluídos pacientes estáveis sob o ponto de vista clínico e de enfermagem, mas fisicamente autossuficientes quanto ao atendimento das necessidades humanas básicas; nos cuidados intermediários (27-39 pontos) estão os pacientes estáveis sob o ponto de vista clínico, com parcial dependência das ações de enfermagem para o atendimento das necessidades humanas básicas; nos cuidados semi-intensivos (40-52 pontos) estão os pacientes crônicos, estáveis sob o ponto de vista clínico, porém, com total dependência das ações de enfermagem quanto ao atendimento das necessidades humanas básicas e, nos cuidados intensivos (53-65 pontos) estão os pacientes graves, com risco iminente de vida ${ }^{(3)}$.

Para compor as três avaliações no banco de dados desse estudo, utilizou-se a primeira avaliação realizada após a internação, a avaliação realizada em período médio de internação e a última avaliação realizada antes da alta hospitalar. Para o 
tratamento das informações foi obtido um escore médio das três avaliações e, sobre este escore, foram geradas as análises apresentadas. As demais avaliações foram descartadas da análise.

A análise dos dados teve como abordagem inicial a estatística descritiva com a distribuição de frequências simples e relativa, bem como as medidas de dispersão. Para a comparação de proporções entre as respostas de uma mesma variável, foi utilizado o teste Qui-quadrado, levando em consideração a distribuição teórica de homogeneidade entre as categorias comparadas. Para avaliar a associação entre duas variáveis qualitativas foi implementado o teste Qui-quadrado de Pearson, assim como a correção de continuidade e o Teste Exato de Fisher por simulação de Monte Carlo.

Os dados receberam tratamento estatístico por meio do software SPSS 17.0 (Statistical Package to Social Sciences for Windows). O projeto foi aprovado pelo Comitê de Ética em Pesquisa da PUCRS pelo Parecer CEP nº 1.315/09.

\section{RESULTADOS}

A amostra foi composta de 161 pacientes, avaliados no ano de 2010. Do total de pacientes, 59,6\% eram do sexo feminino, com média de idade de 81,5 $( \pm 8,44)$ anos (variação de 60 a 99 anos). A faixa etária prevalente foi a de 80 a 100 anos com 67 idosos $(60,2 \%)$ e a média de tempo de permanência hospitalar foi de 13,4 dias ( $\pm 9,7)$ com variação de 2 a 68 dias e prevalência de 6 a 10 dias (38,5\%). Dos idosos investigados, $145(90,1 \%)$ tiveram como desfecho da internação a alta hospitalar e 9,9\% (16) dos idosos foram a óbito.

A Tabela 1 apresenta a média da pontuação e a pontuação mínima e máxima de cada indicador crítico do SCP, conforme momento de avaliação. Os indicadores críticos motilidade, locomoção, cuidados corporais e eliminações foram os que obtiveram maior média, independente do momento da avaliação. Na média geral dos indicadores críticos também houve pequena variação nas três avaliações realizadas.

Tabela 1 - Média e o desvio padrão da pontuação de cada indicador crítico do SCP, conforme momento de avaliação.

Porto Alegre-RS, 2011

\begin{tabular}{|c|c|c|c|c|c|c|}
\hline \multirow{2}{*}{ Indicadores } & \multicolumn{2}{|c|}{ Pontuação Primeira Avaliação } & \multicolumn{2}{|c|}{$\begin{array}{l}\text { Pontuação Avaliação } \\
\text { Intermediária }\end{array}$} & \multicolumn{2}{|c|}{ Pontuação Última Avaliação } \\
\hline & Média \pm DP & Min -Máx & Média \pm DP & Min -Máx & Média \pm DP & Min -Máx \\
\hline Estado Mental e Nível de Consciência & $2,3 \pm 1,2$ & $1-5$ & $2,4 \pm 1,2$ & $1-5$ & $2,4 \pm 1,2$ & $1-5$ \\
\hline Oxigenação & $1,4 \pm 0,7$ & $1-4$ & $1,4 \pm 0,7$ & $1-3$ & $1,3 \pm 0,7$ & $1-4$ \\
\hline Sinais Vitais & $1,0 \pm 0,0$ & $1-1$ & $1,0 \pm 0,0$ & $1-1$ & $1,0 \pm 0,1$ & $1-3$ \\
\hline Nutrição & $2,6 \pm 1,1$ & $1-5$ & $2,5 \pm 1,1$ & $1-5$ & $2,5 \pm 1,2$ & $1-4$ \\
\hline Motilidade & $3,3 \pm 1,4$ & $1-5$ & $3,3 \pm 1,5$ & $1-5$ & $3,3 \pm 1,5$ & $1-5$ \\
\hline Locomoção & $3,7 \pm 1,3$ & $1-5$ & $3,5 \pm 1,5$ & $1-5$ & $3,6 \pm 1,4$ & $1-5$ \\
\hline Cuidados Corporais & $3,9 \pm 1,3$ & $1-5$ & $3,8 \pm 1,4$ & $1-5$ & $3,9 \pm 1,3$ & $1-5$ \\
\hline Eliminações & $3,4 \pm 1,2$ & $1-5$ & $3,4 \pm 1,3$ & $1-5$ & $3,4 \pm 1,3$ & $1-5$ \\
\hline Terapêutica & $1,9 \pm 0,1$ & $1-4$ & $1,8 \pm 0,7$ & $1-4$ & $1,7 \pm 0,8$ & $1-4$ \\
\hline Educação à Saúde & $1,4 \pm 0,8$ & $1-5$ & $1,4 \pm 0,9$ & $1-5$ & $1,5 \pm 1,0$ & $1-5$ \\
\hline Comportamento & $1,3 \pm 0,8$ & $1-4$ & $1,4 \pm 0,9$ & $1-5$ & $1,3 \pm 0,8$ & $1-5$ \\
\hline Comunicação & $2,2 \pm 1,4$ & $1-5$ & $2,3 \pm 1,5$ & $1-5$ & $2,3 \pm 1,5$ & $1-5$ \\
\hline Integridade Cutaneomucosa & $1,8 \pm 1,1$ & $1-5$ & $1,9 \pm 1,0$ & $1-5$ & $2,0 \pm 1,1$ & $1-5$ \\
\hline Média geral dos indicadores críticos & $30,2 \pm 9,2$ & $13-49$ & $29,9 \pm 9,4$ & $13-47$ & $30,1 \pm 9,4$ & $13-52$ \\
\hline
\end{tabular}

Tabela 2 - Categorias de cuidado, conforme momento de avaliação e média das três avaliações. Porto Alegre-RS, 2011

\begin{tabular}{lccc}
\hline Sistema de Classificação de Pacientes & $\begin{array}{c}\text { Primeira Avaliação* } \\
\mathbf{n}=\mathbf{1 6 1}\end{array}$ & $\begin{array}{c}\text { Avaliação Meio* } \\
\mathbf{n}=\mathbf{1 6 1}\end{array}$ & $\begin{array}{c}\text { Última Avaliação* } \\
\mathbf{n}=\mathbf{1 6 1}\end{array}$ \\
\hline Cuidados mínimos & $50(31,1)$ & $52(32,3)$ & $62(38,5)$ \\
Cuidados intermediários & $86(53,4)$ & $85(52,8)$ & $72(44,7)$ \\
Cuidados semi-intensivos & $25(15,5)$ & $24(14,9)$ & $27(16,8)$ \\
Cuidados intensivos & - & - & $-2,4)$ \\
\hline
\end{tabular}

*Valores apresentados da forma $n$ (\%), com percentuais obtidos com base no total da amostra $(n=161)$. 
Tabela 3 - Distribuição absoluta e relativa para o sexo, faixa etária, tempo de permanência e desfecho da internação, segundo as categorias de cuidado. Porto Alegre-RS, 2011.

\begin{tabular}{|c|c|c|c|c|}
\hline \multirow{2}{*}{ Variáveis } & \multicolumn{3}{|c|}{ Classificação Conforme média das três avaliações * } & \multirow[b]{2}{*}{$\mathbf{P}$} \\
\hline & Cuidado Mínimo & Cuidado Intermediário & Cuidado Semi- intensivo & \\
\hline \multicolumn{5}{|l|}{ Sexo } \\
\hline Masculino & $22(38,6)$ & $33(39,3)$ & $10(50,0)$ & \multirow{2}{*}{$0,642+$} \\
\hline Feminino & $35(61,4)$ & $51(60,7)$ & $10(50,0)$ & \\
\hline \multicolumn{5}{|l|}{ Faixa Etária } \\
\hline 60 a 80 anos & $33(57,9)$ & $27(32,1)$ & $04(20,0)$ & \multirow{2}{*}{$0,001+$} \\
\hline 80 a 100 anos & $24(42,1)$ & $57(67,9)$ & $16(80,0)$ & \\
\hline \multicolumn{5}{|c|}{ Permanência Hospitalar } \\
\hline Até 5 dias & $10(17,5)$ & $7(8,3)$ & $1(5,0)$ & \multirow{4}{*}{$0,387 \ddagger$} \\
\hline De 6 a 10 dias & $21(36,8)$ & $36(42,9)$ & $5(25,0)$ & \\
\hline De 11 a 15 dias & $11(19,3)$ & $19(22,6)$ & $7(35,0)$ & \\
\hline 16 dias ou mais & $15(26,3)$ & $22(26,2)$ & $7(35,0)$ & \\
\hline \multicolumn{5}{|l|}{ Desfecho } \\
\hline Alta Hospitalar & $57(100,0)$ & $76(90,5)$ & $12(60,0)$ & \multirow{2}{*}{$0,001 \neq$} \\
\hline Óbito & $0(0,0)$ & $8(9,5)$ & $8(40,0)$ & \\
\hline
\end{tabular}

* Valores apresentados na forma n(\%), onde o percentual obtido com base no total de pacientes em cada classificação da soma total; †: Teste Quiquadrado de Pearson; ¥: Teste Exato de Fisher por simulação de Monte Carlo.

A Tabela 2 apresenta a classificação dos idosos internados por categorias de cuidado, na qual a categoria de cuidados intermediários foi a mais prevalente, independente do período de internação.

Na Tabela 3 são apresentados os dados das associações das variáveis do estudo com as categorias de cuidado do SCP. Os idosos na faixa etária de 80 a 100 anos e os que tiveram o óbito como desfecho da internação, mostraram-se estatisticamente associados aos cuidados intermediários e semi-intensivos.

\section{DISCUSSÃO}

Dos aspectos analisados neste estudo, identificou-se que houve pequena variação entre os escores da primeira avaliação, da avaliação intermediária e da última avaliação, com variação de 30,2, 29,9 e 30,1 pontos respectivamente, caracterizando a dependência para os cuidados de enfermagem no nível intermediário (52,2\%). Os pacientes classificados com cuidados mínimos totalizaram 35,4\% dos idosos. Não foi encontrado, na amostra deste estudo, idosos com cuidados intensivos, no entanto, $12,4 \%$ das pontuações da média das três avaliações concentraram-se nos cuidados semi-intensivos (Tabela 1 e 2).

Estes dados diferem dos dados encontrados quanto ao paciente em várias faixas etárias, idosos e não idosos internados em unidade clínica e cirúrgica que identificou prevalência dos pacientes na categoria de cuidados mínimos, sendo $73 \%$ na unidade clínica e $46 \%$ na unidade cirúrgica. Na unidade cirúrgica também foi identificado prevalência de 30\% dos pacientes em cuidados intermediários ${ }^{(7)}$.

Um estudo realizado com idosos internados em unidade clínica identificou prevalência de $36,6 \%$ na categoria de cuidado intermediário, seguida por 33,3\% em cuidados mínimos e 26,0\% em cuidados semi-intensivos ${ }^{(8)}$.

Os dados de pacientes de várias faixas etárias, em comparação com os dados deste estudo e outro específico com idosos $^{(8)}$, são indicativos de que há um agravamento de saúde dos pacientes idosos e que os profissionais envolvidos devem considerar as alterações do envelhecimento que esta clientela pode se mostrar mais dependente dos cuidados de enfermagem.

As variáveis sexo e tempo de permanência não tiveram, neste estudo, valor estatístico significante. Mas a idade, quando avaliada por faixa etária, demonstrou que os idosos na faixa etária de 80 a 100 anos estiveram relacionados com as categorias de cuidado intermediário e semi-intensivo (Tabela 3), demonstrando que com o avançar da idade o idoso se torna mais dependente em todos os indicadores críticos de cuidado, tornando-se um desafio para a equipe de enfermagem prestar uma assistência de qualidade. Este desafio deve ser percebido e entendido pelos gestores no planejamento de ações ligadas a processos de cuidar e dimensionamento de pessoal.

Quanto à alta ou ao óbito do idoso, como desfecho da internação hospitalar, os pacientes classificados em cuidados mínimos tiveram, em sua totalidade, o desfecho como alta 
hospitalar (100\%). Dos pacientes com cuidados intermediários $90,5 \%$ tiveram como desfecho a alta hospitalar e 9,5\% o óbito. Dado extremamente relevante encontrado neste estudo é o de que $40 \%$ dos idosos classificados com cuidados semi-intensivos tiveram o óbito como desfecho (Tabela 3). A ampliação da longevidade no contexto brasileiro traz consigo o aumento da prevalência de doenças crônicas e dos agravos à saúde e, consequentemente, a presença maior de idosos nos ambientes hospitalares. Este aspecto deve ser considerado na assistência prestada pela enfermagem, que recebe um contingente cada vez maior de pacientes idosos com faixas etárias elevadas e com graves complicações à sua saúde.

Estudo de perfil de morbidade e de mortalidade de pacientes idosos internados revelou prevalência de óbitos de 20,3\% naqueles com 80 anos ou mais e de $11,7 \%$ na faixa etária de 60 a 79 anos. O mesmo estudo sugere que deve ser dado ênfase à avaliação de cuidados prestados a este segmento populacional, para promover maior conhecimento acerca da efetividade dos cuidados prestados ${ }^{(9)}$.

Este estudo identificou que os indicadores críticos com pontuações mais elevadas foram motilidade, locomoção, cuidados corporais e eliminações (Tabela 1). Tal verificação indica para um volume aumentado do trabalho da equipe de enfermagem, incluindo a transferência do paciente do leito para a poltrona, higiene corporal, troca de fraldas, dentre outros. Este aspecto deve ser levado em consideração no dimensionamento da equipe de enfermagem desta unidade, com especial atenção para a escala diária de cuidados.

Este método de avaliação do grau de dependência do paciente ainda é pouco utilizado nas instituições de saúde, o que pode minimizar o entendimento dos padrões de avaliação e as possibilidades de comparação e compreensão dos mecanismos de cuidado e suas repercussões para a qualidade da assistência e a prevenção de agravos à saúde dos pacientes. Identificou-se em um estudo ${ }^{(10)}$ que $44 \%$ dos enfermeiros dimensionam pela gravidade, $30 \%$ pelo número de pacientes e apenas 4\% aplicam a Resolução Cofen 189/96 que utiliza o SCP para o cálculo de pessoal de enfermagem.

\section{CONSIDERAÇÕES FINAIS}

Identificou-se que os idosos internados nesta unidade possuem dependência parcial das ações de enfermagem para o atendimento das necessidades humanas básicas (cuidados intermediários), e que um percentual expressivo de pacientes crônicos, embora estáveis sob o ponto de vista clínico, apresentam total dependência das ações de enfermagem quanto ao atendimento das necessidades humanas básicas, sendo caracterizado como cuidados semi-intensivos. O estudo demonstra que o uso da escala de classificação de pacientes de Perroca e Gaidzinski, pode proporcionar conhecimentos específicos acerca do cuidado no processo de envelhecer, possibilitando a sistematização da assistência de enfermagem ao idoso hospitalizado.

\section{REFERÊNCIAS}

1. Santos F, Rogenski NMB, Baptista CMC, Fugulin FMT. Sistema de classificação de pacientes: proposta de complementação do instrumento de Fugulin et al. Rev Latino-Am Enferm 2007;15(5):103-8.

2. Laus AM, Anselmi ML. Caracterização dos pacientes internados nas unidades médicas e cirúrgicas do HCFMRP-USP, segundo o grau de dependência em relação ao cuidado de enfermagem. Rev Latino-Am Enferm 2004;12(4):643-9.

3. Perroca MG, Gaidzinski RR. Sistema de classificação de pacientes: construção e validação de um instrumento. Rev Esc Enferm USP 1998;32(2):153-68.

4. Perroca MG. Instrumento para classificação de pacientes: opinião de usuários e análise de indicadores de cuidado. Rev Esc Enferm USP 2008;42(4):656-4.

5. Perroca MG, Gaidzinski RR. Instrumento de classificação de pacientes de Perroca: teste de confiabilidade pela concordância entre avaliadores - correlação. Rev Esc Enferm USP 2002;36(3):245-52.
6. Ministério da Saúde. (Brasil). Portaria Ministerial $\mathrm{n}^{\circ}$. 2528, de 19 de outubro de 2006. Dispõe sobre a Política Nacional de Saúde da Pessoa Idosa. Diário Oficial da União 19 out 2006.

7. Araújo VB, Perroca MG, Jericó MC. Variabilidade do grau de complexidade assistencial do paciente em relação à equipe de enfermagem. Rev Latino-Am Enferm 2009;17(1):34-9.

8. Sales FM, Santos I. Perfil de idosos hospitalizados e nível de dependência de cuidados de enfermagem: identificação de necessidades. Texto \& Contexto Enferm 2007;16(3):495-502.

9. Amaral ACS, Coeli CM, Costa MCE, Cardoso VS, Toledo ALA, Fernandes CR. Perfil de morbidade e de mortalidade de pacientes idosos hospitalizados. Cad Saúde Pública 2004;20(6):1617-26.

10. Vigna $C P$, Perroca MG. Utilização de sistema de classificação de pacientes e métodos de dimensionamento de pessoal de enfermagem. Arq Ciênc Saúde 2007;14(1):8-12. 